\title{
Contexto y aprendizaje: el papel de la interacción en diferentes tipos de tareas
}

\author{
PILAR LACASA \\ PILAR HERRANZ \\ Universidad Nacional de Educación a Distancia
}

\begin{abstract}
Resumen
Este trabajo analiza la incidencia del contexto material y social en el aprendizaje de niños entre cinco y ocho años. Se asume que puede existir una interacción entre estas dos dimensiones del contexto. Se presentan datos relacionados con el aprendizaje en una tarea causal y otra lógico-matemática considerando la eficacia de la interacción del niño con el adulto y/o los iguales. La muestra está formada por 96 niños, distribuidos en un grupo de control y dos grupos experimentales. Se realizan análisis cuantitativos y cualitativos. Los resultados muestran que la interacción individual con el adulto resulta especialmente eficaz en la tarea causal y, sobre todo, en los niños que tenian un nivel más bajo al comenzar el proceso de aprendizaje. Por otra parte, la interacción con los iguales produce mejores resultados en ambas tareas cuando los niños interactúan con otros niños más hábiles en la tarea. Finalmente, se observan diferencias en la eficacia de las estrategias de instrucción que utiliza el adulto en función del tipo de tarea.
\end{abstract}

Palabras clave: Contexto, Aprendizaje, Toma de conciencia, Análisis de tareas.

\section{Context and learning. The role of interaction in different kind of tasks}

\section{Abstract}

This paper analyzes the role of social and material context in leaming. Context must be defined along two dimensions; the physical and the social-cultural environment. The main objective is to analize the impact of interaction in the learning process; athe grasp of consciousness* in different kinds of tasks. The subjects of the study were 96 Spanish children from 5 to 8 years of age. They were assigned either to a control group or to one of the several experimental groups. Each child was tested individually in a pre-test and a post-test. Children in the experimental groups also participated in a training session and all sessions were tape-recorded. Results suggest different effects of interaction in the learning process depending on another children interact with the adult individually or with a peer. Finally, differences in the efficiency of instruction strategies used by the adult in function of the type of task involved were observed.

Keywords: Learning, Grasp of Consciousness, Task Analysis.

Agradecimientos: Esta investigación ha sido financiada con cargo a la convocatoria de ayudas a la investigación del CIDE (1985-1988). Una primera versión de este trabajo fue presentada en Budapest, 15-19 junio, 1988, «Third European Conference on Developmental Psychology". Agradecemos a D. Villuendas su contribución a esta investigación, también a J. García Madruga sus comentarios a la primera versión de este trabajo.

Dirección del autor: UNED, Facultad de Psicología, Departamento de Psicología Evolutiva, Ciudad Universitaria, s/n, 28040, Madrid.

Original recibido: septiembre 1988. Revisión recibida: diciembre 1988. Aceptado: diciembre 1988. 


\section{INTRODUCCION}

Una primera aproximación a la idea de contexto permite distinguir en él un complejo nudo de interacciones entre el entorno físico y el entorno socio-cultural, dimensiones múltiples que se entrelazan pero que resultan difíciles de analizar conjuntamente. Diferentes investigaciones han estudiado la incidencia del contexto en el aprendizaje considerando, exclusivamente, unos $u$ otros de esos aspectos. Aludir al contexto puede significar, por ejemplo, definirlo a partir de la interacción que el niño mantiene con otras personas más capaces y en relación con las cuales aprende. Pero también consideran el contexto, más o menos explícitamente, quienes ponen el acento en los componentes materiales de la tarea a la que se enfrenta un sujeto cuando resuelve un problema.

En este trabajo se analiza el papel del contexto en el aprendizaje aceptando que aquel debe ser definido, al menos, desde esta doble dimensión, es decir, el niño aprende a resolver problemas interactuando con un entorno físico y sociocultural. Algunos planteamientos neopiagetianos y neovygotskyanos, que constituyen el marco teórico de esta investigación, permitirán comprender mejor las cuestiones precisas que en ella se plantean y que se agrupan en torno a tres núcleos temáticos cuya convergencia justificaremos en esta introducción. El primer núcleo de cuestiones a los procesos cognitivos del niño que serán objeto de estudio, analizaremos la toma de conciencia de la acción propia en situaciones de aprendizaje. En segundo lugar nos referiremos también a la importancia de factores procedentes del entorno físico, las características de la tarea condicionan, el conocimiento infantil; desde este punto de vista nos preguntamos qué estrategias utiliza el niño para abordar tareas relacionadas con problemas causales y lógico matemáticos. Por último, un tercer campo de problemas, se refiere a la interacción del niño con el adulto y/o con los iguales. Más concretamente, nuestro objetivo es analizar la incidencia de la interacción sobre el proceso de aprendizaje de la toma de conciencia de la acción propia en diferentes tipos de tareas.

Varias razones justifican un análisis de los procesos de toma de conciencia aunque hemos de reconocer que este término, tal como es utilizado por Piaget (1974a; 1974b; 1975), puede ser una fuente de confusiones. Tomar conciencia significa para Piaget reconstruir la propia acción en el plano de la representación e implica una verbalización. Imaginemos a un niño que resuelve un problema práctico, es posible diferenciar tres planos en su conducta: «hacer", «saber hacer" y «saber que se sabe». Es en este último plano donde se sitúa la toma de conciencia. Otros autores aluden a procesos cognitivos muy próximos a éste aunque con diferente terminología, por ejemplo, autorregulación (Brown y col. 1983), metaprocesos (KarmiloffSmith, 1984) o, incluso, conocimiento metaconceptual (Carey, 1985). Estas investigaciones han mostrado las relaciones de estos procesos con el desarrollo cognitivo y se acepta, por ejemplo, que una mayor o menor eficacia del niño en procesos de este tipo puede contribuir a explicar las diferencias que existen entre expertos y novatos en el aprendizaje o en situaciones de solución de problemas. En nuestra opinión, por otra parte, un análisis de los procesos de toma de conciencia parece especialmente adecuado para determinar en qué medida las aportaciones de Piaget y Vygotsky 
resultan compatibles y se complementan (Lacasa \& Villuendas, 1988; Lacasa, en prensa). Piaget, a diferencia que Vygotsky, no analizó nunca cuáles son las relaciones entre la verbalización y la acción, ni tampoco en qué medida la verbalización puede facilitar al niño la ejecución de la tarea, investigaciones muy recientes (Brown, 1987) señalan la necesidad de profundizar en este tema.

Aludiremos ahora a la importancia del contexto relacionado con el tipo de tarea a la que el niño se enfrenta. Trabajos anteriores, realizados por una de nosotras, han mostrado la incidencia de las peculiaridades de la tarea en los procesos de toma de conciencia. Por ejemplo, este proceso resultaba más fácil a los niños de preescolar y ciclo inicial en tareas lógicomatemáticas que en causales o motoras (Lacasa y col., 1985a); en las tareas lógico-matemáticas se descubrían diferentes niveles de dificultad en función de que los niños manipularan unos materiales u otros (Lacasa y col., 1985b). Desde otra perspectiva, distintas investigaciones han considerado también la necesidad de analizar estos procesos en relación con diferentes tipos de tareas (Karmiloff-Smith, 1984; Krupa, Selman, Jaquette, 1985). Finalmente señalaremos que, desde otro marco teórico, quienes han realizado análisis más detallados de las tareas y de los procesos cognitivos relacionados con ella proceden de la psicología que se ha inspirado en el procesamiento de la información (Siegler y Klahr, 1982; Siegler, 1985).

Nos referiremos, por último, al tercero de los aspectos presentes en este trabajo, la interacción del niño con el adulto y/o los iguales. El problema del aprendizaje, analizado a partir del concepto de «zona de desarrollo próximo» que aporta Vygotsky (1978) nos conduce a considerar la incidencia del contexto social en el desarrollo y en el aprendizaje, dos aspectos que, por otra parte, resultan difícilmente separables desde esta perspectiva teórica. Podemos diferenciar dos líneas de investigación en los trabajos actuales que se han inspirado en ese concepto. Por una parte, los que se han realizado en la escuela de Ginebra (Doise, 1983; Doise y Mugny, 1981; Perret Clermont, 1984) considerando el papel de la interacción con los iguales como factor del desarrollo; en ellos se revisa, más concretamente, el lugar del conflicto sociocognitivo como factor capaz de producir cambios en el conocimiento cuando los niños interactúan con otros del mismo, menor o superior nivel de desarrollo. En nuestra opinión, estos estudios asumen que los resultados obtenidos en investigaciones relacionadas con el aprendizaje proporcionan, desde un planteamiento microgenético, un conocimiento más profundo de lo que ocurre en el desarrollo en un nivel macrogenético. Es decir, los cambios en la conducta que se producen en situaciones de aprendizaje, y por tanto en períodos de tiempo relativamente breves, serian similares a los que tienen lugar a través del desarrollo infantil. Por otra parte, trabajos recientes de la psicología anglosajona han analizado las diferencias que pueden producirse en situaciones de aprendizaje (Ellis y Rogoff, 1986; Brown y Reeve, 1987) o de solución de problemas (Wersch, 1985; Kontos, 1983) cuando los niños interactúan con el adulto o con los iguales (Forman y Cazden, 1985). Se acepta, desde esta perspectiva, que adulto o el compañero más capaz proporcionan soporte y ayuda, en situaciones de tutoria y colaboración, en cuanto que introducen una mejor aproximación a la tarea, es decir, se acercan a ella a través de estrategias más complejas y útiles que las del niño, pero y que progresivamente èl irá 
adoptando. En estas investigaciones, a diferencia de lo que ocurre en los estudios de la escuela de Ginebra, se aportan análisis detallados no sólo de los resultados del proceso de aprendizaje, sino también de las estrategias que tanto el que aprende como el que enseña van introduciendo.

\section{OBJETIVOS}

Partiendo de los presupuestos del apartado anterior, este estudio pretende:

1. Determinar las relaciones que pueden establecerse entre dos tipos de tareas, en un proceso de enseñanza-aprendizaje orientado a facilitar la toma de conciencia. Este objetivo puede concretarse en los siguientes puntos.

1.1. Analizar la relación entre ambas tareas considerando especialmente la secuencia temporal del proceso de aprendizaje.

1.2. Analizar el rendimiento de los sujetos en el aprendizaje en función de la presencia o ausencia de progreso en ambas tareas.

2. Determinar el papel del adulto y de los iguales, considerando el nivel de los niños que interactúan, en el aprendizaje en relación con tareas lógico-matemáticas y causales. Especificaremos también este objetivo.

2.1. Analizar la incidencia de las estrategias de instrucción que utiliza el adulto, en situaciones de tutoría, sobre el aprendizaje del niño.

2.2. Analizar la interacción del niño con los iguales y con el adulto en situaciones de colaboración y su incidencia en el aprendizaje.

\section{METODO}

\section{Sujetos}

La muestra está formada por 96 niños y niñas de Preescolar y Ciclo Inicial que pertenecían a dos colegios públicos de Madrid. Se tomaron al azar 32 niños de cada grado escolar, cuya media de edad era de 5,5 (Preescolar), 6,9 (1. ${ }^{\circ}$ de EGB) y 7,6 (2. ${ }^{\circ}$ de EGB). Los niños se distribuyeron en un grupo de control $(\mathrm{N}=24)$ y dos grupos experimentales, atendiendo al tipo de interacción social que mantendrian en la sesión de intervención: un grupo , en que los niños interactuarían individualmente con el adulto $(\mathrm{N}=24)$ y otro grupo en el que la interacción con el adulto se haría por parejas $(\mathrm{N}=48)$. En cada uno de estos grupos existía una distribución proporcional de los niños en función del curso escolar al que pertenecían. Posteriormente, con el fin de precisar la incidencia del nivel de desarrollo de los ninos que interactuaban por parejas en el aprendizaje, se constituyeron tres subgrupos atendiendo al nivel de desarrollo que los niños del grupo citado manifestaron en el pretest realizado en relación con cada una de la tareas.

\section{Procedimiento}

Los niños asignados a los grupos experimentales participaron en tres sesiones en relación con cada una de las tareas, a saber, un pretest y un postest que realizaron individualmente y una sesión de intervención en la cual, y en función del grupo al que habían sido asignados, los niños interactua- 
ron individualmente con un adulto, o con un adulto y otro niño del mismo, inferior o superior nivel de desarrollo. El adulto, en la sesión de entrenamiento, debía intervenir con el fin de facilitar al niño la toma de conciencia; si bien en principio se pretendía controlar la incidencia del conflicto sociocognitivo en el aprendizaje, la propia dinámica de las sesiones de intervención indujo al adulto a introducir diferentes estrategias de acuerdo con su propio criterio. Dichas estrategias fueron categorizadas y analizadas, posteriormente, en función de su eficacia. Los niños del grupo de control realizaron sólo las sesiones individuales de pretest y postest.

Las dos tareas fueron propuestas por Piaget (1974), pero en este trabajo se introdujeron algunas modificaciones, tanto en el pretest y el postest como en las sesiones de intervención. En otros trabajos hemos presentado una descripción detallada de estas tareas que ahora resumimos (Lacasa \& Villuendas, 1988).

\section{Pretest $y$ postest}

Tarea causal. Se trata del juego de la pulga que se presenta a los niños a través de cuatro situaciones. Sit. 1: Sobre un tapete los niños deben hacer saltar una pequeña ficha dentro de una caja ayudándose de otra mayor. Sit. 2: La tarea es la misma pero se realiza directamente sobre la mesa. Sit. 3: La consigna es similar pero los materiales varian, el niño debe hacer saltar dentro de la caja y sobre el mismo tapete que se utilizaba en la situación 1, una ficha más gruesa y una caja de cerillas. Sit. 4: La tarea es similar a la que se presenta en la situación 3 pero ahora ha desaparecido el tapete. En cada una de las situaciones, una vez que el niño ha realizado la tarea, el adulto provoca la toma de conciencia pidiendo al niño que describa y explique su acción y el resultado de ésta verbalmente y por medio de un dibujo que sirve de apoyo a la verbalización.

Tarea lógico matemática. Tanto en pretest como en el postest se propone al niño ordenar en función de su anchura seis cartulinas blancas, cada una con una mancha de diferente color en uno de sus extremos. Estos elementos tienen idéntica longitud y diferente anchura, varian entre sí gradualmente en un centímetro, la más ancha es de $16 \mathrm{~cm}$ y la más estrecha de $11 \mathrm{~cm}$. Para provocar la toma de conciencia el adulto propone al niño la descripción y explicación de su actividad, en presencia de los elementos - mientras realiza la acción y una vez que la ha finalizado- y en ausencia de ellos -dando instrucciones al adulto para que sea éste quien construya la serie.

\section{Sesión de Intervención}

Los niños realizaron tanto la tarea lógico matemática como la causal. En ambos casos se introdujeron modificaciones en las dos tareas que se orientaron en una triple dirección: a) El adulto presentó las tareas de un modo mucho más flexible. b) Se introdujeron cambios en los materiales orientados a facilitar la comprensión del proceso. c) El adulto utilizó diferentes estrategias de intervención, que revisaremos a continuación, para facilitar la toma de conciencia. 


\section{4}

\section{Análisis realizados}

A partir de los protocolos verbales que incluían transcripciones completas de todas las sesiones, se han llevado a cabo dos tipos de análisis. En primer lugar, un análisis cualitativo, orientado a determinar diferentes niveles de complejidad en el rendimiento de los sujetos en ambos tipos de tareas; dicho análisis se apoya en lo que se refiere a la asignación de puntuaciones, en los trabajos de Damon y Killen (1982) y Perret Clermont (1984). En segundo lugar, y a partir de los resultados obtenidos en el análisis cualitativo se ha realizado un análisis cuantitativo orientado a determinar las relaciones que es posible establecer entre el rendimiento de los sujetos en ambos tipos de tareas, considerando la incidencia de diferentes situaciones de interacción social en el proceso de aprendizajè.

\section{CODIFICACION Y PUNTUACIONES}

Una primera revisión de los protocolos permitió fijar un código de categorías a partir de las cuales se analizaron tres dimensiones de la conducta. Una primera dimensión se refiere al proceso de toma de conciencia del niño, considerando sus descripciones y explicaciones verbales en las dos tareas y en cada una de las tres sesiones a través de las cuales se investigó el proceso de aprendizaje. Los códigos utilizados en el análisis de estas respuestas y los criterios cuantitativos de valoración han sido descritos minuciosamente por una de nosotras en otro lugar (Lacasa y Villuendas, 1988). Las otras dimensiones de conducta se refieren a la interacción que los niños mantienen con los iguales y/o con el adulto en la sesión de intervención. En relación con este aspecto se consideraron, por una parte, diferentes situaciones de colaboración entre quienes participaban de la situación $y$, por otra, las estrategias que introducía el adulto, orientadas a facilitar la toma de conciencia.

\section{Descripciones y explicaciones verbales de la tarea}

Dos comentarios previos a la exposición de los criterios de análisis de las tareas son necesarios. En primer lugar, que las categorías de análisis que se proponen no se refieren a la acción del niño, ya que en ambas tareas el niño lograba el éxito con relativa facilidad, sino a las descripciones y explicaciones de su actividad que surgían cuando el niño tomaba conciencia de los procedimientos que habían intervenido en ella. En segundo lugar, que estos criterios de análisis se aplicaron en el pretest, en el postest y en la sesión de intervención.

\section{Tarea causal}

La tabla I muestra las categorías de acuerdo con las cuales se analizaron las respuestas de los niños en la tarea causal. Dichas categorías son el resultado de un análisis muy minuicioso de las respuestas de los niños y a partir del cual se diferenciaron 50 «respuestas tipo". En relación con dichas "respuestas tipo" se delimitaron las actuales categorías atendiendo a dos criterios: a) El contenido de las respuestas que puede hacer referencia a cuatro aspectos del fenómeno y a los que hemos denominado "Dominios de 
la tarean y que aparecen en la tabla I. Por ejemplo, el niño puede referirse a la ficha que recibe el impulso y que debe saltar dentro de la caja, pero. unas veces alude a su movimiento (Dom. 1) y otras a sus características (Dom. 2); en otros casos el niño alude a su acción (Dom. 3) o al efecto que ésta produce sobre el objeto (Dom. 4). b) El nivel de complejidad de las respuestas. Como puede observarse en la tabla I, las respuestas de los niveles más bajos son animistas o incluyen características no relevantes para la explicación causal del fenómeno que pueden, incluso, deformar la realidad; posteriormente, se limitan a describir lo que observan y, finalmente, en los niveles superiores no sólo describen sino que intentan explicar y para ello consideran uno o varios aspectos del fenómeno.

Los protocolos verbales de todas las sesiones fueron analizados de acuerdo con este código. El $20 \%$ de los protocolos fue codificado por dos jueces. Para evaluar la fiabilidad del acuerdo entre los jueces se aplicó el coeficiente de acuerdo de Scott (Anguera, 1983) en cada uno de los protocolos seleccionados, los valores obtenidos oscilaban entre $=0,40 \mathrm{y}=1$. Se asignó a cada niño una puntuación, en función de su nivel, en cada uno de los dominios de la tarea; ese nivel coincidía con el de su respuesta más compleja. Se le asignó también una puntuación global, considerando los niveles alcanzados en cada dominio, que expresaba su rendimiento en la explicación de la tarea.

\section{Tarea lógico matemática}

Los criterios de análisis que aparecen en la tabla II habían sido ya determinados en anteriores investigaciones (Lacasa, P. López y P. Llantada, 1985; Lacasa y Villuendas, 1988). Se introdujeron, sin embargo, algunas modificaciones. Como puede observarse en la tabla II, las respuestas de los niños aluden, directa o indirectamente, a las estrategias que han utilizado en la construcción de la serie y que revelan las relaciones que ellos son capaces de establecer entre los elementos. Dichas estrategias fueron definidas por nosotras a partir de un análisis de las «respuestas tipo» que aparecen también en la tabla II. En esta tarea se diferenciaron también varios grados de complejidad en las respuestas y, a partir de ellos, se asignó a los sujetos un determinado nivel y una puntuación.

\section{Interacción social}

La interacción social representa una de las dimensiones del contexto que son objeto de estudio en este trabajo. Conviene recordar que nuestro objetivo es determinar la influencia de la interacción del niño con el adulto y con los iguales en el proceso de aprendizaje; desde esta perspectiva se comprenderá mejor que fue necesario fijar dos tipos de categorías orientadas a analizar la interacción social en la sesión de intervención. Por una parte, aquellas que permitieran describir cuál era el grado de colaboración real que existía entre ellos y, por otra parte, cuáles eran las estrategias específicas del adulto orientadas a provocar y facilitar la toma de conciencia.

\section{Situaciones de colaboración}

Una primera revisión de los protocolos verbales permitió, de nuevo, determinar un conjunto de categorías que aparecen en la tabla III y de acuer- 
TABLA I

Categorías de análisis. Tarea causal

\begin{tabular}{|c|c|c|c|c|c|}
\hline Dominios & Nivel I & Nivel II & Nivel III & Nivel IV & Nivel V \\
\hline $\begin{array}{l}\text { DOMINIO "A" } \\
\text { Referencia el movimiento } \\
\text { de la ficha u objeto pasivo }\end{array}$ & $\begin{array}{l}\text { Interpretación animista } \\
\text { del movimiento }\end{array}$ & $\begin{array}{l}\text { Denominación simple del } \\
\text { movimiento }\end{array}$ & $\begin{array}{l}\text { Alusión al efecto del } \\
\text { movimiento }\end{array}$ & $\begin{array}{l}\text { Alude al movimiento de la } \\
\text { ficha y a su dirección }\end{array}$ & $\begin{array}{l}\text { Interpretación causal del } \\
\text { movimiento }\end{array}$ \\
\hline $\begin{array}{l}\text { DOMINIO "B» } \\
\text { Referencia a las caracteris- } \\
\text { ticas del objeto que recibe } \\
\text { el impulso }\end{array}$ & $\begin{array}{l}\text { Referencia a característi- } \\
\text { cas no relevantes del ob- } \\
\text { jeto }\end{array}$ & $\begin{array}{l}\text { Referencias a las caracte- } \\
\text { rísticas relevantes del ob- } \\
\text { jeto, pero manteniendo } \\
\text { contradicciones }\end{array}$ & $\begin{array}{l}\text { Determinación de las } \\
\text { características relevantes } \\
\text { del objeto }\end{array}$ & $\begin{array}{l}\text { Distingue las característi- } \\
\text { cas relevantes del objeto } \\
\text { en función del movimien- } \\
\text { to }\end{array}$ & $\begin{array}{l}\text { Diferenciación del movi- } \\
\text { miento en función de las } \\
\text { características relevantes } \\
\text { del objeto }\end{array}$ \\
\hline $\begin{array}{l}\text { DOMINIO "C" } \\
\text { Relaciones entre el sopor- } \\
\text { te y el movimiento del ob- } \\
\text { jeto }\end{array}$ & $\begin{array}{l}\text { Alusiones aunque irrele- } \\
\text { vantes al papel que en el } \\
\text { movimiento ocupa el so- } \\
\text { porte }\end{array}$ & $\begin{array}{l}\text { Alusiones, aunque contra- } \\
\text { dictorias al papel que ocu- } \\
\text { pan los diferentes soportes } \\
\text { en el movimiento }\end{array}$ & $\begin{array}{l}\text { Referencias a los dife- } \\
\text { rentes soportes y a sus } \\
\text { relaciones con la forma } \\
\text { del movimiento }\end{array}$ & $\begin{array}{l}\text { Trata de justificar el mo- } \\
\text { vimiento según la presen- } \\
\text { cia o ausencia del soporte }\end{array}$ & $\begin{array}{l}\text { Explica la relación entre el } \\
\text { soporte y el movimiento } \\
\text { que se desencadena según } \\
\text { el objeto en juego }\end{array}$ \\
\hline $\begin{array}{l}\text { DOMINIO «D" } \\
\text { Relaciones entre la acción } \\
\text { del sujeto y el objeto }\end{array}$ & & $\begin{array}{l}\text { El niño se refiere simple- } \\
\text { mente a la acción que ha } \\
\text { realizado }\end{array}$ & $\begin{array}{l}\text { Se refiere a la forma de } \\
\text { ejecutar la acción }\end{array}$ & $\begin{array}{l}\text { Se refiere a la acción y a la } \\
\text { forma de transmitir el im- } \\
\text { pulso }\end{array}$ & $\begin{array}{l}\text { Explica el movimiento del } \\
\text { objeto aludiendo a la pre- } \\
\text { sión que ejerce y a la po- } \\
\text { sición de la ficha }\end{array}$ \\
\hline
\end{tabular}


TABLA II

Categorias de análisis. Tarea lógico-matemática

\begin{tabular}{|c|c|c|c|}
\hline Estrat. & Nivel & $\begin{array}{l}\text { Descripción de } \\
\text { la respuesta }\end{array}$ & $\begin{array}{l}\text { Categorías de } \\
\text { la observación }\end{array}$ \\
\hline 1 & I & $\begin{array}{l}\text { - Los niños responden con deno- } \\
\text { minaciones arbitrarias que no } \\
\text { introducen el orden entre los } \\
\text { elementos }\end{array}$ & $\begin{array}{l}\text { 1. Porque lo ví. Porque toca. } \\
\text { 2. Esta, esa, la que está debajo. } \\
\text { 3. Poniendo uno y otro. }\end{array}$ \\
\hline 2 & I & $\begin{array}{l}\text { - Respuestas referidas al color } \\
\text { adicional de las cartulinas para } \\
\text { expresar el orden }\end{array}$ & $\begin{array}{l}\text { 4. Roja. Morada. } \\
\text { 5. Establece comparaciones entre } \\
\text { la gama de colores. }\end{array}$ \\
\hline 3 & II & $\begin{array}{l}\text { - Respuestas que aluden al tama- } \\
\text { no de las cartulinas, pero con ca- } \\
\text { rácter absolutizador. Etiquetado }\end{array}$ & $\begin{array}{l}\text { 6. Justifica la relación con índi- } \\
\text { ces perceptivos. } \\
\text { 7. «La gruesa. La pequeña. La } \\
\text { grande. }\end{array}$ \\
\hline 4 & III & $\begin{array}{l}\text { - Respuestas que aluden a una } \\
\text { cierta jerarquización entre los } \\
\text { elementos }\end{array}$ & $\begin{array}{l}\text { 8. La primera. La segunda... La } \\
\text { última. } \\
\text { 9. La más alta y la más ancha. }\end{array}$ \\
\hline 5 & IV & $\begin{array}{l}\text { - Respuestas que encadenan la re- } \\
\text { lación de los elementos de la se- } \\
\text { rie estableciendo parejas o tríos }\end{array}$ & $\begin{array}{l}\text { 10. Compara las tarjetas dos a } \\
\text { dos. } \\
\text { 11. La más grande de las pequeñas } \\
\text { (considera dos o tres del to- } \\
\text { tal). } \\
\text { 12. La más pequeña de las tres que } \\
\text { quedan. }\end{array}$ \\
\hline 6 & IV & $\begin{array}{l}\text { - Respuestas que establecen rela- } \\
\text { ciones entre cuatro o cinco ele- } \\
\text { mentos de la serie }\end{array}$ & $\begin{array}{l}\text { 13. Sugiere comparar varias: «Mí- } \\
\text { delas». } \\
\text { 14. Comparando más de tres: } \\
\text { «Unas son más grandes que } \\
\text { otras.» } \\
\text { 15. Más grande que las otras tres } \\
\text { que quedan. }\end{array}$ \\
\hline 7 & V & $\begin{array}{l}\text { - Respuestas que establecen rela- } \\
\text { ción transitiva entre los elemen- } \\
\text { tos de la serie }\end{array}$ & $\begin{array}{l}\text { 16. La más mayor de todas. La } \\
\text { más pequeña. } \\
\text { 17. Mayor que la que va detrás. } \\
\text { 18. Menor que la anterior. }\end{array}$ \\
\hline 8 & VI & $\begin{array}{l}\text { - Respuestas que establecen rela- } \\
\text { ciones transitivas en el interior } \\
\text { de la serie y encadenan ordena- } \\
\text { ción entre los elementos de la } \\
\text { serie en doble dirección. }\end{array}$ & $\begin{array}{l}\text { 19. Indica al adulto que reúna to- } \\
\text { das las cartulinas para com- } \\
\text { pararlas. } \\
\text { 20. La más estrecha de las que } \\
\text { quedan. }\end{array}$ \\
\hline
\end{tabular}

do con las cuales se analizó, posteriormente, el tipo de interacción que mantenían entre sí los participantes en la situación. Una breve justificación de cómo se llegaron a fijar esas categorías es necesaria. En la revisión de los protocolos verbales, referidos a la tarea causal, se observó que en muchas ocasiones los participantes de la situación conversaban entre sí aludiendo a aspectos del fenómeno físicio que muy poca o ninguna relación tenían entre sí. Una situación frecuente era, por ejemplo, que el adulto planteara al niño una cuestión pero que éste respondiera algo, quizá una idea a la que aludia constantemente, que no tenía ninguna relación con lo que se le preguntaba. Se observó también que algo semejante ocurría cuando dos niños, en presencia del adulto, conversaban entre sí. En otras situaciones, por el 
contrario, todos o algunos de los participantes «dialogaban y colaboraban realmente». Estas primeras observaciones nos hicieron pensar que no era suficiente considerar en las sesiones de intervención la presencia del adulto o la de los iguales, como a primera vista pudiera pensarse; debía analizarse también qué grado de colaboración se mantenía ya que sus efectos en relación con el progreso del niño en el aprendizaje serían importantes. Se determinó, por tanto, un código que permitiera delimitar esa colaboración; desde ese punto de vista se consideró como un criterio válido para diferenciar las situaciones, que aparecen en la tabla III, el hecho de que quienes interactuaban se refirieran a los mismos o diferentes dominios de la tarea. Somos conscientes de que el código de observación propuesto es incompleto, entre otras razones porque atiende sólo al análisis de las respuestas verbales, pero, en principio, permitirá controlar cuál, entre las diferentes situaciones es más eficaz cuando se trata de facilitar la toma de conciencia. Es importante señalar, además, que estas categorías sólo pudieron ser aplicadas en el caso de la tarea causal y que ello fue debido como se comprenderá fácilmente, al criterio utilizado para diferenciarlas - la referencia a los diferentes dominios de la tarea. Creemos necesario, en trabajos futuros, fijar categorías que permitan analizar esta dimensión de la conducta cuando el sujeto realiza otra clase de tareas.

TABLA III

Colaboración en tareas causales

\begin{tabular}{ll}
\hline \multicolumn{1}{c}{ Situaciones } & \multicolumn{1}{c}{ Tipo de interacción } \\
\hline 1. No hay colaboración & - El niño, su compañero y/o el adulto se refieren a di- \\
ferentes dominios de la tarea. \\
2. Pares & - El niño y su compañero se refieren al mismo domi- \\
nio de la tarea. & - El niño y el adulto se refieren al mismo dominio de \\
3. Adulto/niño & la tarea. \\
4. Adulto/pares & El adulto y los dos niños se refieren al mismo domi- \\
& nio de la tarea. \\
\hline
\end{tabular}

\section{Estrategias de intervención del adulto}

Dichas estrategias, que aparecen en la tabla IV, se determinaron a partir de un análisis de los protocolos de las sesiones de intervención. Posteriormente todas las estrategias utilizadas por el adulto fueron codificadas de acuerdo con esas categorías. El $20 \%$ de los protocolos fueron analizados por dos jueces; para evaluar la fiabilidad del acuerdo se aplicó un índice de porcentaje de acuerdo (Anguera, 83) para cada una de las categorías. Los resultados fueron: estrategias 1,4, y $5, F=100 \%$; estrategia 6 , $\mathrm{F}=83 \%$; estrategia $3, \mathrm{~F}=63 \%$; estrategia $2, \mathrm{~F}=53 \%$.

Una lectura detallada de esa tabla permite observar que las estrategias se orientan en una triple dirección: a) Establecer relaciones entre los distintos aspectos de las tareas (Est. 1, Est. 2 y Est. 3) lo cual facilitaria los procesos de codificación del niño. b) Introducción del dibujo, lo cual supondrá la utilización de, al menos, dos tipos de representación por parte del niño (Est. 4 y Est. 5). c) Superación de conflictos (Est. 6). 


\section{9}

TABLA IV

Estategias del adulto

Conducta que

el adulto

sugiera al niño
Tarea Causal

Tarea

lógico-matemática
1. Describir y/o explicar El resultado de su acción

2. Relacionar aspectos Aquellos dominios de la tarea presentes de la tarea. que el niño percibe.

3. Relacionar aspectos Se pide al niño que prevea lo no presentes de la tarea

4. Dibujar

5. Explicar el dibujo

6. Superar dificultades
La trayectoria de la ficha y su caída en la caja en las diferentes situaciones de la tarea.

Puede referirse al de una situación a comparar varios dibujos

Cuando el niño realiza observaciones incorrectas el adulto le provoca contradicciones.
Cómo ha construido la serie. Comparar entre si diferentes tarjetas o una de ellas con el resto.

El adulto construye la serie de acuerdo con las instrucciones del niño, pero éste no tiene ante si los elementos.

La serie de cartulinas que ha construido.

Justifica por qué ha realizado ese dibujo y las relaciones entre las cartulinas.

El adulto sugiere acciones, por ejemplo, superponer los elementos, que facilitan la comparación cuando el niño percibe las diferencias incorrectamente.

\section{ANALISIS Y DISCUSION DE LOS RESULTADOS}

El análisis se orientó en la dirección de los objetivos propuestos. En primer lugar, se determinaron las correlaciones entre ambos tipos de tareas a través del proceso de aprendizaje. Para ello se consideró el rendimiento de los sujetos en cada una de las sesiones y, además, el grado de progreso en las dos tareas establecido a partir de las diferencias entre el pretest y el postest. A partir de estos resultados es posible examinar las relaciones entre el pensamiento causal y lógico matemático (Piaget, 1975; Krupa, Selman, Jaquette, 1985) en procesos de toma de conciencia; el hecho de analizar la toma de conciencia (Piaget, 1974a) ha permitido, además, plantear la discusión en un doble plano: por una parte, considerar, desde una perspectiva microgenética, procesos que tienen lugar en el desarrollo a un nivel macrognético, especialmente la reconstrucción de la acción en el plano de la representación; por otra parte, establecer si es posible facilitar, a través del aprendizaje, procesos de autorregulación.

En segundo lugar, con el fin de conocer la posible interrelación entre los diferentes factores del contexto, se llevó a cabo un análisis de las situaciones de interacción del niño con el adulto o con los iguales para determinar si dicha interacción tenía el mismo efecto en ambas tareas. Se tuvieron en cuenta dos aspectos, por una parte, las estrategias que utiliza el adulto en situaciones de tutoría y, por otra, la presencia o ausencia de colaboración entre quienes interactúan. La discusión de estos resultados puede plantearse también en un doble nivel: el primero, el papel de la interacción en el desarrollo cognitivo (Doise, 1983) y, el segundo, la incidencia del adulto en el aprendizaje (Wertsch, 1985; Ellis y Rogoff, 1986). 


\section{El proceso de aprendizaje en tareas lógico matemáticas y causales}

\section{Relaciones entre las dos tareas}

Con el fin de determinar la relación entre ambas tareas, considerando la secuencia temporal del proceso de enseñanza-aprendizaje, se calculó el coeficiente de correlación de Spearman entre las puntuaciones obtenidas por los niños en cada una de las tareas para cada una de las sesiones (pretest, entrenamiento y postest). En este análisis se obtuvieron correlaciones independientes en cada uno de los grupos de sujetos que habían recibido un entrenamiento diferente en la sesión de intervención. Los valores obtenidos en estas correlaciones aparecen en la figura 1. Como puede observarse allí las correlaciones aumentan a través del proceso, entre el pretest y el postest, pero hay diferencias entre ellas en función del grupo al que pertencen los sujetos. Se obtuvieron correlaciones bajas entre el rendimiento de los niños en las dos tareas, durante el pretest, en todos los grupos (Control, $\mathrm{r}=.22$; Interacción individual con el adulto, $\mathrm{r}=.07$; Interacción por parejas con el adulto, $r=-.07)$. El valor de las correlaciones aumentó en todos los grupos en la sesión de intervención, pero ese aumento fue mucho más importante cuando niños actuaban individualmente con el adulto $(r=.38 ; p<0,05)$. Puede observarse, por último, que para el mismo grupo esta correlación desciende en el postest y se obtiene una correlación negativa $(r=-.27)$. En el caso de una interacción por parejas con el adulto la correlación aumenta gradualmente (entrenamiento, $r=.20$; postest, $\mathrm{r}=.35, \mathrm{p}<0,05)$ a través del proceso de aprendizaje.

Una interpretación de estos resultados sugiere diferentes efectos de la interacción sobre el proceso de aprendizaje, en función de que los niños se relacionen con el adulto individualmente o por parejas. Podemos aceptar, en principio, que la interacción por parejas produce mejores resultados; la existencia en este grupo de un aumento gradual de las correlaciones sugiere un progreso de los niños en ambas tareas a través del proceso de aprendizaje. Estos datos son similares a los obtenidos en el grupo de control, también aquí existe un aumento gradual entre pretest y postest; estos niños no realizaron sesión de entrenamiento. Este dato es explicable, quizá, porque el hecho de realizar dos veces la misma tarea produce una mejora en el rendimiento. Los datos que resultan más difíciles de explicar son los obtenidos cuando el niño interactúa individualmente con el adulto, en este caso la correlación positiva alta entre las tareas en la sesión de entrenamiento y la correlación negativa en el postest sugiere, por una parte, que los efectos del entrenamiento no se mantienen en alguna de las tareas; por otra parte, es posible pensar que el adulto puede facilitar en el niño los procesos de codificación (Siegler, 1985) o la estructuración más eficaz de la tarea (Ellis y Rogoff, 1986), pero que el niño no es capaz de realizar el mismo proceso, en alguna de las tareas, cuando actúa solo.

Con el fin de obtener una información más detallada de lo que ocurría cuando teníamos en cuenta las diferencias de nivel entre los niños que interactuaban por parejas, constituimos a partir de este grupo tres subgrupos. Uno de ellos formado por los niños que interactuaron con el adulto y otro de su mismo nivel $(N=14)$, otro grupo formado por los niños que interactuaron con otro de nivel superior $(N=17) y$, finalmente, un terce- 
FIGURA 1

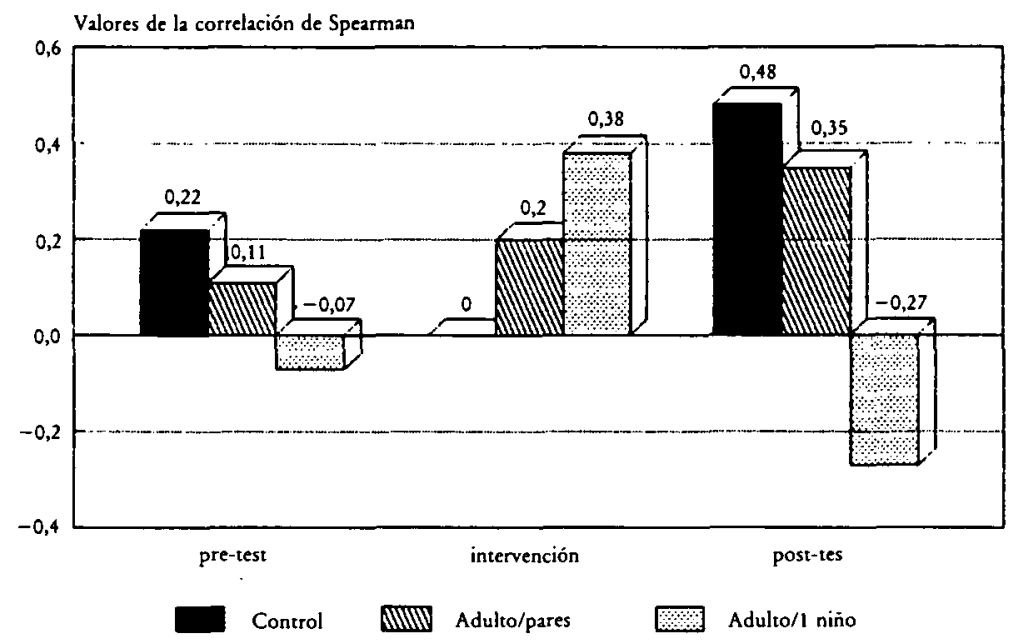

Relaciones tarea lógico-matemática y causal

ro con niños que interactuaron con otro de nivel inferior $(N=17)$. De nuevo obtuvimos las correlaciones entre las tareas para cada uno de los grupos y en cada una de las sesiones. Los resultados aparecen en la figura 2. Como puede observarse aquí las correlaciones aumentaron sensiblemente en el postest en todos los grupos, pero sobre todo en los niños que tenían un menor nivel que su compañero (pares -), es decir, en aquellos que interactuaron con otro de nivel superior (pretest, $r=0,18$; post-test, $r=0,52$, $p<0,05)$. La correlación aumentó también en el postest en el grupo de niños que actuaron con otros de su mismo nivel (pares =) (prestest, $r=-0,1$; postest, $r=0,31$ ). La correlación que aumentó menos fue la obtenida en el postest en el grupo de niños con mayor nivel que su par (pares + ), es decir, que interactuaron con otro de menor nivel (pretest, $r=0.08$; postest, $r=0,24$ ). Estos datos muestran que la situación que favorece una correlación mayor entre ambas tareas, considerando que se mantiene en el postest, es la interacción de los pares con el adulto y teniendo en cuenta, además, que los más favorecidos por esa interacción eran los que interactuaron con pares de mayor nivel.

\section{El aprendizaje en la tarea lógico-matemática y en la tarea causal}

Estos resultados orientaron el análisis de datos hacia una revisión más profunda del proceso de aprendizaje, considerando el progreso que se babia producido en ambas tareas por separado. Para ello se determinó, el porcentaje de sujetos que habían progresado entre el pretest y el postest en las dos tareas y para cada uno de los grupos. Los resultados aparecen en la figura 3. No se obtuvieron diferencias significativas entre los grupos en la tarea causal, $\mathrm{X}_{2}(3 \times 2)=1.524, \mathrm{GL}=2$, ni en la lógico-matemática, $\mathrm{X}_{2}$ $(3 \times 2)=2.942, \mathrm{GL}=2$. Destaca, sin embargo, el hecho de que en la tarea causal progresan más los niños que interactúan individualmente con el adulto $(67 \%)$, mientras que en la lógico matemática apenas hay diferencias 
Figura 2

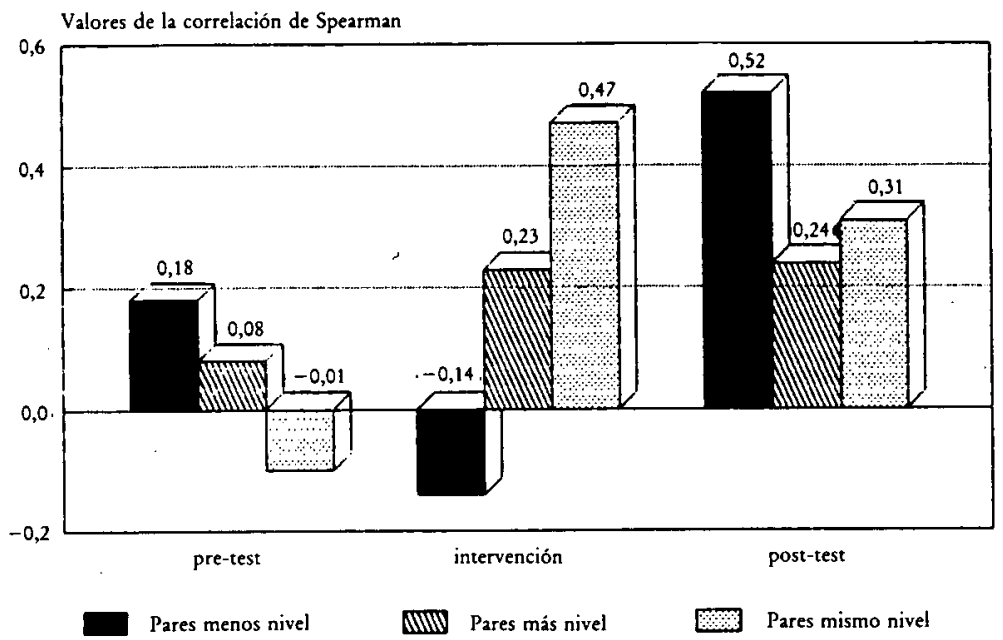

Relaciones tarea lógico-matemática y causal

entre los niños que interactúan con el adulto por parejas $(74 \%)$ o individualmente $(71 \%)$. Una posible explicación de la eficacia de la interacción individual con el adulto en la tarea causal sería el mayor grado de dificultad de esta tarea; en uno de nuestros trabajos anteriores (Lacasa y col., 1985a) encontramos que los niños de esta edad tenían un menor nivel en la comprensión de los fenómenos causales que en las tareas lógico-matemáticas.

FIGURA 3a

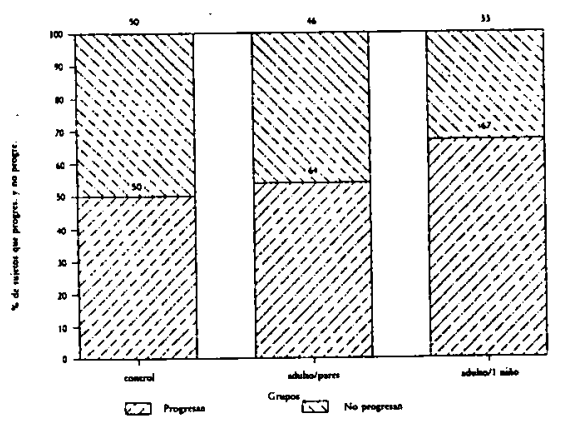

Tarea causal
Figura $3 b$

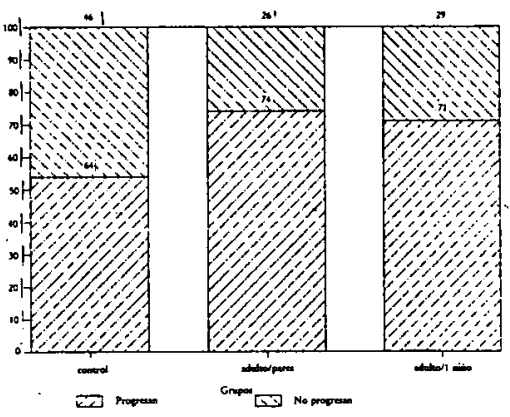

Tarea lógico-matemática

Con el fin de analizar con más detalle el progreso que se había producido a consecuencia del aprendizaje en ambas tareas se realizaron dos nuevos análisis. El primero orientado a controlar el "efecto suelo», es decir, la incidencia del nivel que tenian niños en el pretest sobre su proceso de aprendizaje en ambas tareas. El segundo para determinar el efecto de la interacción con los iguales en el aprendizaje, en ambas tareas por separado, considerando el nivel de los niños interactuantes. 
La figura 4 muestra los valores obtenidos en la correlación de Spearman entre el nivel que los niños tenían en el pretest y el progreso que se produjo en la prueba. Dicho progreso se determinó asignando a cada sujeto una puntuación que expresaba la diferencia entre el nivel alcanzado en el pretest y el logrado en el postest. Como puede observarse, los valores de las correlaciones, en todos los grupos y para ambas tareas, son negativos, ello significa que los sujetos con un menor nivel en el pretest fueron los que progresaron más. Las correlaciones resultan significativas en todos los casos $(p<, 05)$ salvo en la tarea lógico-matemática en el grupo en que los niños interactuaron individualmente con el adulto $(r=-0,21)$. Este dato sugiere de nuevo diferencias en la eficacia de la interacción en función de la tarea. La interacción individual con el adulto favoreció mucho más a los niños que tenían menor nivel en el pretest en la tarea causal que en la lógico-matemática.

Con el fin de analizar, como ya hemos indicado, el efecto sobre el aprendizaje del nivel de los niños que interactúan por parejas con el adulto, se

FIGURA 4

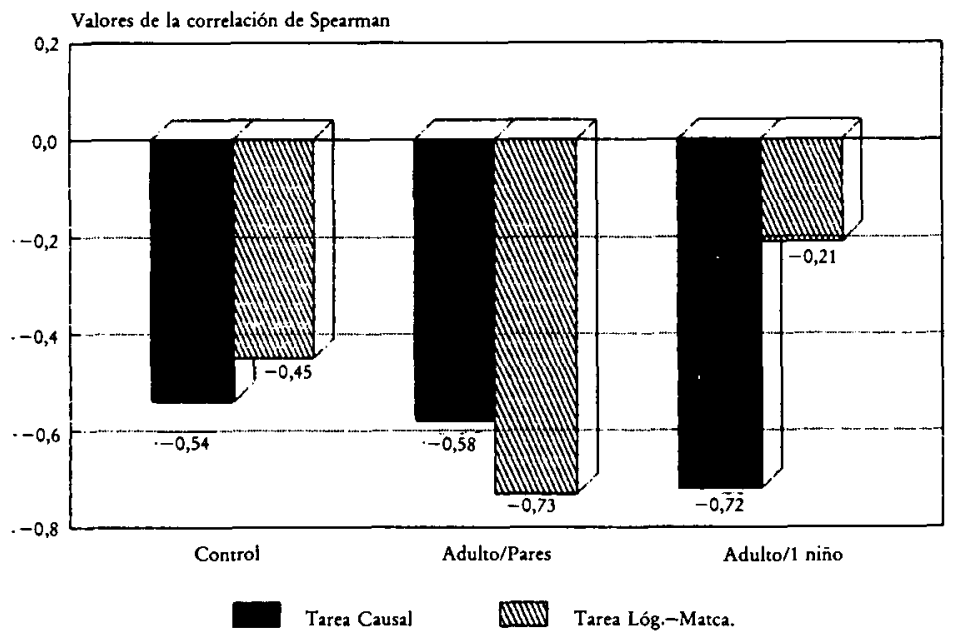

Nivel de pretest y progreso

determinó el porcentaje de sujetos que progresaron y no progresaron en cada uno de los tres subgrupos. Es decir, cuando los pares tenían el mismo nivel (pares $=$ ), cuando los niños tenían mayor nivel que su compañero ( $\mathrm{pa}$ res + ) o, finalmente, cuando su nivel era menor (pares -). Los resultados de este análisis aparecen en la figura 5.

En las dos tareas se obtienen diferencias significativas entre los grupos. En la tarea causal, $\mathrm{X}_{2}(3 \times 2)=14,47, \mathrm{GL}=2, \mathrm{p}<0,01$; en la tarea lógico-matemática, $X_{2}(3 \times 2)=10,12, G L=2, p<0,01$. Se observa claramente que el mayor progreso se produce, en ambas tareas, entre los niños que interactuaron con el adulto y otro de mayor nivel (causal, $92 \%$; lógico-matemática, $88 \%$ ). Por el contrario, existen diferencias entre las tareas 
en relación con el número de niños que progresan en los otros grupos. Cabe destacar, por una parte, que si los niños interactuantes son del mismo nivel progresan más en la tarea lógico-matemática ( $88 \%$ ) que en la causal $(50 \%)$. Señalaremos, por otra parte, que apenas progresaron los niños que interactúan con otro de nivel inferior, ello es especialmente claro en la tarea causal $(24 \%)$, y menos evidente en el caso de la tarea lógico-matemática $(47 \%)$.

FIGURA 5a.

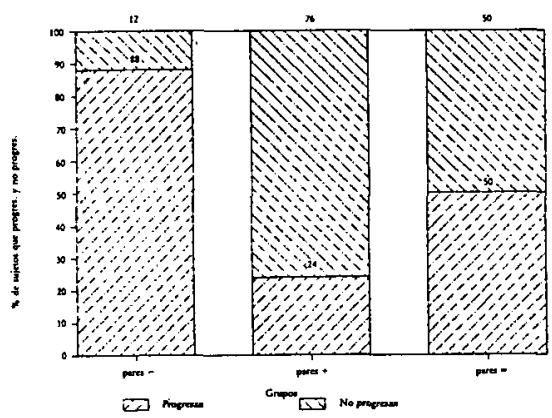

Tarea causal
FIGURA $5 b$

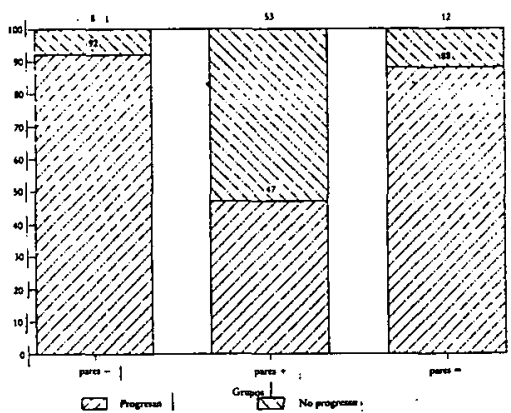

Tarea lógico-matemática

Algunos comentarios a propósito de la relación entre el pensamiento lógico matemático y causal en diferentes situaciones de interacción social, son necesarios como conclusión de esta primera parte del análisis. Piaget (1975) había propuesto un desarrollo relativamente paralelo de ambos tipos de conocimiento dirigido, en último término, por la adquisición de estructuras lógicas cada vez más complejas. Investigaciones de Krupas, Selman y Jaquette (1985) mostraron una correlación entre las explicaciones científicas y el pensamiento operatorio tal como se manifiesta en tareas lógico matemáticas, pero estos autores sugieren, sin embargo, que en determinadas ocasiones el razonamiento lógico parece ir por delante. En términos generales nuestros resultados apoyarían las conclusiones de este trabajo. Es necesario, en cualquier caso, insistir en la especificidad de las tareas que son, en definitiva, una de las dimensiones del contexto al que Piaget tuvo muy poco en cuenta. Los resultados muestran, por otra parte, que el aprendizaje puede facilitar la autorregulación (Brown y col. 1983), considerándola como un proceso de construcción de representaciones de la propia actividad ejercida sobre los objetos en situaciones de solución de problemas. Por último, y por los que se refiere a las situaciones de interacción social, la interacción con otros niños resulta especialmente eficaz en aquellos que tienen un nivel menor que su compañero, pero, por el contrario, es muy poco útil para los que tienen un nivel mayor. Esto nos hace pensar que es más importante, en el proceso de aprendizaje, aportar al niño una ayuda orientada a mejorar la estructuración y codificación de la tarea (Ellis y Rogoff, 1986) que la existencia de posibles conflictos sociocognitivos (Doise, 1983) a los que el niño se enfrenta cuando tiene ante sí soluciones erróneas como las que puede proponer, por ejemplo, su compañero de menor nivel. 


\section{Interacción en situaciones de tutoría y colaboración}

Un análisis de los propios de la sesión de intervención permitió obtener resultados relacionados con la eficacia de las distintas situaciones de interacción sobre el aprendizaje (Forman y Cazden, 1985). Dichas situaciones podían ser de dos tipos: a) de tutoría, relacionadas con las diferentes estrategias utilizadas por el adulto, para facilitar la toma de conciencia; b) de colaboración, relacionadas con el tipo de información que intercambian quienes interactúan; hablamos de colaboración cuando el adulto y/o los niños se refieren a los mismos aspectos de la tarea, es decir, existe un diálogo real entre ellos.

\section{Estrategias de intervención del adulto}

Con vistas a conocer la eficacia de las estrategias utilizadas por el adulto en las dos tareas se determinó el número total de respuestas que el niño dio en cada estrategia, diferenciando aquellas que suponían un progreso en relación con el pretest. Se obtuvo el porcentaje de respuestas, respecto del total en cada estrategia, que suponían un progreso. Estos porcentajes aparecen en la figura 6 . Como puede observarse allí una misma estrategia puede tener distinta eficacia en función del tipo de tarea. Algunas de las estrategias más eficaces en la tarea causal no lo fueron en la lógico matemática.

FiguRA 6

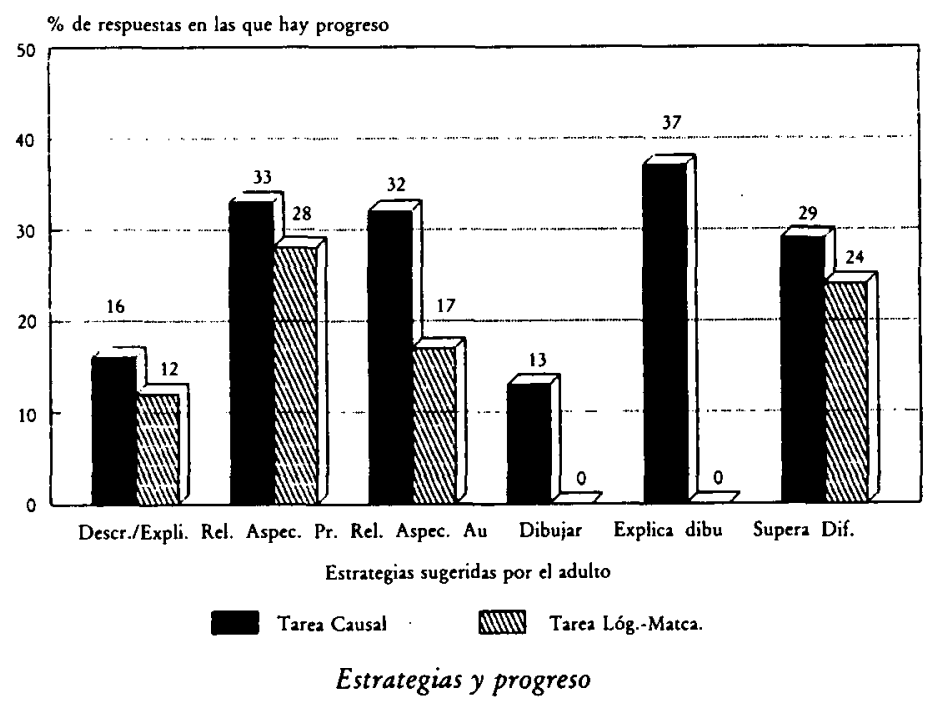

Veremos aquellas estrategias en las que se obtienen diferencias significativas entre los porcentajes referidos al número de respuestas que suponen un progreso, cuando la estrategia se utiliza en una u otra tarea. Es importante resaltar, por otra parte, que las distintas estrategias resultan más eficaces en la tarea causal, de nuevo insistiremos en que este dato puede estar relacionado con la mayor dificultad que para los niños tiene esa tarea. En cualquier caso, serán necesarios nuevos trabajos para precisar la eficacia de las estrategias al menos teniendo en cuenta dos aspectos: por una parte, 
la existencia de diversos sistemas de representación (Karmiloff-Smith, 1984), por ejemplo, el dibujo o la verbalización; por otra, la estructuración y codificación de la tarea (Siegler, 1985; Ellis y Rogoff, 1986). Aludiremos ya a la eficacia de algunas estrategias de un modo más concreto. Para obtener la significación estadística de las diferencias entre ellas se aplicó un contraste de hipótesis para dos proposiciones. Unicamente se obtuvieron diferencias significativas en relación con la eficacia de las estrategias en dos de ellas. La explicación del propio dibujo es la estrategia que da mejores resultados en la tarea causal ( $37 \%$ de respuestas que suponían un progreso frente al nivel del niño en el pretest), mientras que en la tarea lógico matemática, los niños no dieron ninguna respuesta que significara un progreso $(\mathrm{z}=1,87 ; \mathrm{p}<, 05)$. Los resultados son bastante similares cuando el niño se limita a dibujar el fenómeno, sin que el adulto pida ninguna explicación, en este caso el $13 \%$ de las respuestas suponen un progreso en la tarea causal y no hay ninguna respuesta que suponga un progreso en la lógico matemática, en este caso no hubo diferencias significativas estadísticamente. Cuando el adulto sugiere al niño que establezca relaciones entre los aspectos no presentes de la tarea, lo cual supone aportarle una ayuda en sus procesos de codificación, se obtienen diferencias significativas entre los porcentajes de respuestas que suponen un progreso en ambas tareas (32\%, tarea causal y $16 \%$, tarea lógico-matemática, $\mathrm{z}=2,45, \mathrm{p}=, 005)$.

\section{Situaciones de colaboración y aprendizaje}

Un análisis de los protocolos de la sesión de intervención sugirió que la presencia del adulto o de un igual cuando los niños habían de resolver la tarea no siempre iba acompañada de una interacción real entre ellos. Las categorías de análisis utilizadas en la tarea causal permitieron determinar que existia colaboración cuando quienes dialogaban se referían al mismo dominio de la tarea, es decir, la pregunta y la respuesta aludían al mismo aspecto del fenómeno. Para conocer la relación entre las situaciones de colaboración real y el progreso de los niños se obtuvieron los valores de la correlación de Spearman entre la frecuencia de respuestas que significaban un progreso frente al pretest y la frecuencia de situaciones en las que había existido una colaboración entre el adulto y/o los pares. Se obtuvieron correlaciones en cada uno de los subgrupos. Los resultados aparecen en la figura 7.

Como puede observarse en esa figura existen diferencias entre los grupos, lo cual es una nueva muestra de que la eficacia de la colaboración con el adulto o con los iguales está muy condicionada por el nivel de los niños que interactúan. Cuando los niños tienen un nivel menor que su compañero, la situación de colaboración que tiene una mayor relación con el progreso que manifestaron en la sesión entrenamiento es la interacción del adulto con un solo niño $(r=65, \mathrm{p}<.05)$. El resultado es muy similar en el grupo de niños que tenían un nivel mayor que el de su compañero ( $\mathrm{r}=$ $0,66, \mathrm{p}<0,05$ ). Por el contrario, cuando los niños tienen el mismo nivel la interacción entre ellos, sin la intervención directa del adulto, muestra una correlación alta con el grado de progreso $(r=0,64, p<0,05)$; en este caso la presencia del adulto no resulta especialmente útil $(r=-0,06)$. Estos datos tienen enorme interés porque pueden significar que el adulto ejerce una 


\section{7}

\section{FIGURA 7}

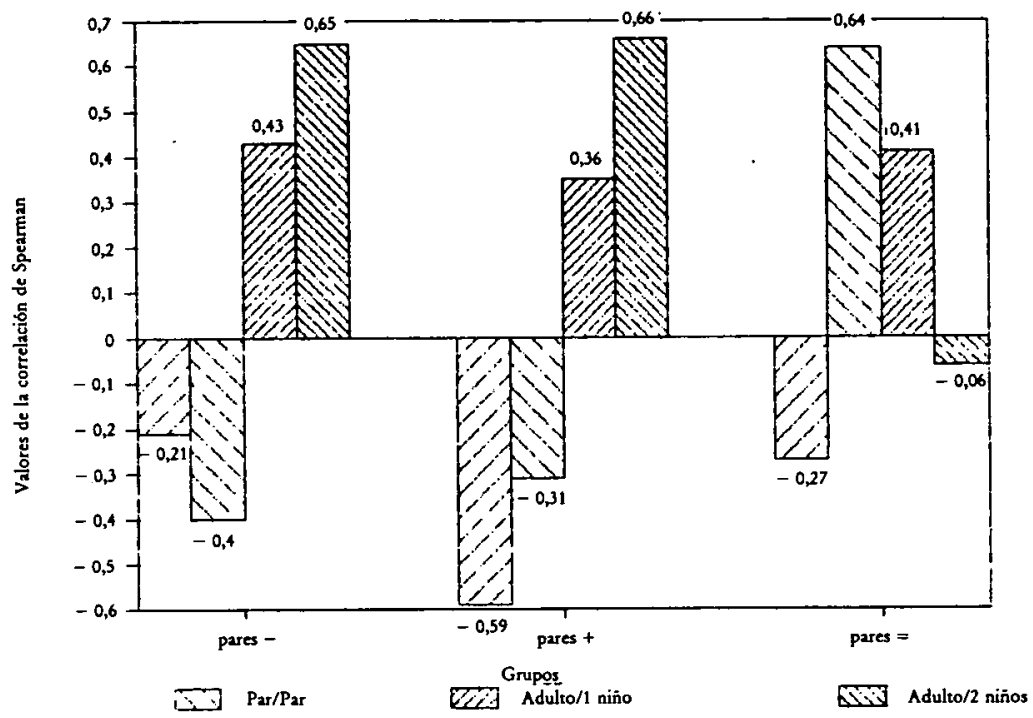

Tarea Causal. Colaboración y progreso

función de puente entre los niños cuando estos tienen diferente nivel, ello sería innecesario en el caso de que ambos fueran del mismo nivel. Estos datos muestran, en principio, que la eficacia de las estrategias y su relación con la tarea puede depender de las ayudas que aporta el adulto para codificar la tarea y de la incidencia de los sistemas de representación.

\section{Conclusión}

Los resultados de este trabajo permiten concluir que los diferentes factores del contexto no inciden en el aprendizaje infantil unos aislados de otros sino que, por el contrario existen interrelaciones entre ellos. El progreso de de los niños está en función no sólo del tipo de tarea sino también de si interactúan con un adulto individualmente o por parejas.

$\mathrm{La}$ interacción individual con el adulto resulta especialmente eficaz en la tarea causal y, sobre todo, para los niños que tenían al comenzar el proceso de aprendizaje un nivel más bajo; por otra parte, también en esta tarea, el adulto parece tener un papel de mediador cuando interactúa con dos niños de diferente nivel, ello les permite un mayor progreso en el aprendizaje. La interacción con los iguales resulta más eficaz en la tarea lógico-matemática. Finalmente, señalaremos que en las dos tareas, esta relación es más útil para los niños que tienen un nivel menor su compañero.

\section{Referencias}

Anguera, T. (1983). Manual de prácticas de observación. México: Trillas.

BROWN, A. L.; BRANSFORD, J. D.; FERRARA, R. A., y CAMPIONE, J. C. (1983). Learning, remembering and understanding. En P. H. Mussen (Ed.): Handbook of Child Psychology. Vol. 3. Cognitive Development. Nueva York: Willey. 
BROWN, A. L. (1987). Metacognition, executive control, self-regulation and other more mysterious mechanisms. En F. E. Weinert y R. H. Kluwe (Eds.): Metacognition, Motivation and Understanding. Hillsdale, N. J.: LEA.

BROWN, A. L., y REEVE, R. A. (1987). Bandwidths of competence: the role of supportive context in learning and development. En L. S. Liben (Ed.): Hillsdale, N. J.: LEA.

CAREY, S. (1985). Are children fundamentally different kinds of thinkers and learners than adults? En S. F. Chipman, J. W. Segal y R. Glaser (Eds.): Thinking and Learning Skills. Research and Open Questions. Vol. 2. Hillsdale, N. J.: LEA.

DAMON, W., y KILLEN, M. (1982). Peer interaction and the process of change in children's moral reasoning. Merrill-Palmer Quarterly, 28, 347-367.

DOISE, W. (1983). Apprentissage, psychologie génétique et psychologie sociale, une transformation de paradigmes. Archives de Psychologie, 51, 17-22.

DOISEW., y MUGNY, G. (1981). Le développement social de l'intelligence. París: Inter Editions.

ELLIS, S., y ROGOFF, B. (1986). Problem solving in children's management of instruction. En E. E. Mueller, C. R. Cooper (Eds.): Process and outcome in peer relationship. Hillsdale, N. J.: LEA.

FORMAN, E. A., y CAZDEN, C. B. (1985). Exploring vygotskian perspetives in education: the cognitive value of peer interaction. En J. V. Wertsch (Ed.): Culture, Communication and Cognition: Vygotskian Perspectives. Cambridge: Cambridge University Press.

KarmiLoff-SmITH, A. (1984). A children's problem solving. M. E. Lamb, A. L. Brown, B. Rogoff (Eds.): Advances in Developmental Psychology. Vol. 3. Hillsdale, N. J.: LEA.

KoNTOS, S. (1983). Adult-Child interaction and the origins of metacognition. Journal of Educational Psychology, 182, 199-244.

Krupa, P. M.; Selman, R., y JaQUETTE, D. (1985). The development of science explanations in children and adolescent: A structural approach. En S. F. Chipman, J. W., Segal y R. Glaser (Eds.): Thinking and Learning Skills. Research and Open Questions. Vol. 2. Hillsdale, N. J.: LEA.

LACASA, P. (1988) (en prensa). The grasp of consciousness in a motor task: Walking on all fours. Archives de Psychologie.

LACASA, P.; P. LÓPEZ, C., y P. LlantAdA, C. (1985a). Conceptualización de la acción propia en niños escolarizados de 4 a 8 años en diferentes tipos de tareas. Revista de Psicología General y Aplicada, 40, 1-24.

LACASA, P.; P. LOPEZ C., y P. Llantada, C. (1985b). Conceprualización de la acción propia y material manipulable en una tarea de seriación. Revista de Psicología General y Aplicada, 40, 1039-1062.

LaCaSa, P., Villuendas, D. (1988). Acción y representación en el niño. Aprendizaje e interacción social. Madrid: Ministerio de Educación y Ciencia. CIDE.

PeRret CleRmont, A. N. (1984). La construcción de la inteligencia en la interacción social. Madrid: Aprendizaje Visor.

PIAGET, J. (1974a). La prise de conscience. Paris: PUF.

PIAGET, J. (1974b). Reussir et comprendre. París: PUF.

Piaget, J. (1975). L'equilibritation des structures cognitives. Probleme central du développement. Paris: PUF.

SIEGLER, R. (1985). Encoding and the development of problem solving. En S. F. Chipman, J. W. Segal y R. Glaser (Eds.): Thinking and Learning Skills. Research and Open Questions. Vol. 2. Hillsdale, N. J.: LEA.

SIEgLER, R., y KLAHR, D. (1982). When do children learn? The relationship between existing knowledge and the acquisition of new knowledge. En R. Glaser (Ed.): Advances in instructional psychology. Vol. 2. Hillsdale, N. J.: LEA.

VyGOTSKI, L. S. (1978). Mind in Society. The Development of Higher Psychological Processes. Cambridge, M.: Harvard University Press.

WERTSCH, J. V. (1985). Adult-Child interaction as a source of self-regulation in children. En S. R. Yussen (Ed.): The Growth of Reflection in Children. Orlando: Academic Press.

\section{Extended summary}

This paper analyzes the role of the context in learning. It is assumed that the context must be defined along two dimensions: the physical and the social-cultural environment. Several Neo-Piagetian and Neo-Vygotskian perspectives which constitute the theoretical framework of this research allow us a better understanding of the essential questions that are posed. They are grouped under three main topics. The first refers to the 
child's cognitive processes that are to be studied: «the grasp of consciousness" in the learning situation is analysed. Others refer to the context; on the one hand, to the cognitive contents related to the characteristics of the task; and on the other hand to adult-child and/or peer interaction.

\section{Objectives}

1. The main purpose is to analyze the incidence of interaction in the learning process of «the grasp of consciousness» in different kinds of tasks.

2. To determine the relationships that are established between two different tasks on a teaching/learning process, orientated towards facilitating "the grasp of consciousness".

3. To determine the role of adults and peers; the achievement level of the children who are interacting is taken into account.

\section{Method}

\section{Subjects}

The subjects under study were 96 Spanish children from 5 to 8 years of age. They were selected from three grades in two state schools: Preschool (infants) $(\mathrm{N}=32$; mean age $=5,5)$, first grade $(\mathrm{N}=32$; mean age $=6,9)$; and second grade $(N=32$; mean age $=7,6)$. Children were assigned either to a control group or to one of several experimental groups wich were formed on the basis of their school grade and developmental level judged by a pre-test.

\section{Procedure}

Each child was tested individually in a pre-test and a post-test. Children in the experimental groups also participated in a training session and all sessions were tape-recorded.

\section{Results}

The results suggest different effects of interaction within the learning process, depending on whether, children interact with the adult individually or with another peer. It can be accepted that the interaction among peers produces better results and an improvement in the two kinds of tasks in the learning process; the causal task and the logical-mathematical task.

In taking into account the differences among peers who are interacting, we argue that the correlations increase appreciatively in the post-test in all the groups, but above all in children who were at a lower level than his/her peer.

With regard to progress, when taking into account the kind of task, we argue that in the causal task the biggest improvement takes place in children interacting with the adult individually $(67 \%)$. Nevertheless, in the logical-mathematical task there is little differences between interacting with an adult $(74 \%)$, and children interacting with an adult individually $(71 \%)$. A possible explanation is that the causal task is harder than the logico-mathematical task. 
When the children who are interacting are at the same level, the progress is more evident in the logical-mathematical task $(98 \%)$ than in the causal task $(50 \%)$. Nonetheless the children who interact with a lower level peer progress very little. This is very clear in the causal task (24\%) and less evident in the logical-mathematical task ( $47 \%)$. 